\title{
Cultural adoption, and validation of the Persian version of the coronary artery disease education questionnaire (CADE-Q): a second-order confirmatory factor analysis
}

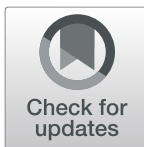

Zahra Marofi ${ }^{1}$, Razieh Bandari ${ }^{2}$, Majideh Heravi-Karimooi ${ }^{3^{*}}$ (D), Nahid Rejeh ${ }^{3}$ and Ali Montazeri ${ }^{4,5^{*}}$

\begin{abstract}
Background: Evaluating knowledge in patients with coronary artery disease requires a specific measure. The aim of the present study was to translate and evaluate the CADE-Q in patients with coronary artery disease in Iran.

Methods: Forward-backward procedure was applied to translate the questionnaire from English into Persian. Then a cross-sectional study was conducted to evaluate psychometric properties of the questionnaire. A sample of patients with coronary artery disease attending to cardiac departments of teaching hospitals affiliated to medical universities in Tehran, Iran completed the 19-item CADE-Q from April to December 2017. Structural validity of CADE-Q was assessed using both exploratory and confirmatory factor analyses. Reliability was examined using Cronbach's alpha coefficient. Stability was evaluated by estimation intraclass correlation coefficient.

Results: In all 500 patients participated in the study. The mean age of patients was 53.63. (SD = 14.36) years, and $57 \%$ were male. The results obtained from the exploratory factor analysis showed a four factor solution (lifestyle habits and exercise, risk factors, diagnosis and treatment, signals \& symptoms and medicine) that jointly explained $48.9 \%$ of the total variance observed. However, the second-order confirmatory factor analysis supported the threefactor solution while convergent and divergent validity were not confirmed. Finally, the Cronbach's alpha coefficient of 0.84 ranging from 0.50 to 0.82 was obtained for the scale and its subscales. In addition, the ICC value of 0.88 showed satisfactory stability for the questionnaire.

Conclusion: The Coronary Artery Disease Education Questionnaire was found to be a multidimensional instrument. The results confirmed the factor structure of the questionnaire with a second-order analysis. Since the convergent and divergent validity of the scale were not confirmed, further assessment is essential to establish fitness of the questionnaire in Iran.
\end{abstract}

Keywords: Coronary artery disease, Psychometric analysis, Reliability, Validity, Knowledge, The CADE-Q

\footnotetext{
* Correspondence: heravi@shahed.ac.ir; montazeri@acecr.ac.ir

${ }^{3}$ Elderly Care Research Center, Faculty of Nursing \& Midwifery, Shahed University, Tehran, Iran

${ }^{4}$ Health Metrics Research Center, Iranian Institute for Health Sciences Research, ACECR, Tehran, Iran

Full list of author information is available at the end of the article
}

(C) The Author(s). 2020 Open Access This article is licensed under a Creative Commons Attribution 4.0 International License, which permits use, sharing, adaptation, distribution and reproduction in any medium or format, as long as you give appropriate credit to the original author(s) and the source, provide a link to the Creative Commons licence, and indicate if changes were made. The images or other third party material in this article are included in the article's Creative Commons licence, unless indicated otherwise in a credit line to the material. If material is not included in the article's Creative Commons licence and your intended use is not permitted by statutory regulation or exceeds the permitted use, you will need to obtain permission directly from the copyright holder. To view a copy of this licence, visit http://creativecommons.org/licenses/by/4.0/ The Creative Commons Public Domain Dedication waiver (http://creativecommons.org/publicdomain/zero/1.0/) applies to the data made available in this article, unless otherwise stated in a credit line to the data. 


\section{Background}

Cardiovascular diseases account for around 16.7 million deaths per year worldwide and it has been estimated that the figure will increase to 23.6 million cases by 2030 [1$3]$. In order to reduce mortality among patients who suffer from cardiac diseases there are many strategies. Of these, cardiac rehabilitation programs are among wellknown strategies. The cardiac rehabilitation programs consist of a set of interventions ranging from physical activity, exercise training, post-operative patient care, the optimization of medical treatment, nutritional counseling, smoking cessation, risk stratification, hypertension management, control of diabetes or dyslipidemia, patient assessment, weight management, aggressive coronary risk factor management, psychosocial counseling, sexual dysfunction, alcohol consumption and stress management $[2,4,5]$. However at the heart of all these programs education is an important component [6-8].

Studies have shown that rehabilitation programs have significant effect in reducing length of hospital stay, improving the quality of life, and patient's performance [9]. In fact such programs could raise patients' level of knowledge and help him/her in making healthy choices in everyday life [10]. Patient education is a process that affects the patients' behavior and changes their knowledge, attitudes, and skills needed to maintain health status [11].

Considering the paramount importance of knowledge in patients with coronary artery disease, various tools have been used to measure their level of knowledge. These tools include the MICRO-Q (Maugeri Cardiac Prevention Questionnaire) [12], the Cardiac Rehabilitation Barriers Scale [13, 14], the Information Need in Cardiac Rehabilitation Scale [15], the Knowledge Questionnaire for Cardiac Failure Patient [16], original version of the Coronary Artery Disease Education Questionnaire, CADE-Q [17], the second version of the CADE-QII [18], and short version of the CADE-Q [19].

Although all this questionnaire are valid instruments, the English version of the CADE-Q seems perform better since it is shorter compared to the other questionnaires and uses four-response categories while most existing questionnaire use true/falls format. As such as part of a project on raising knowledge among patients with coronary artery disease the aim of this study was to translate and validate the English version of the CADE-Q in Iran. Currently no such a questionnaire is available in Iran we aimed.

\section{Methods}

\section{Study design and sample}

This was a cross sectional study on a sample of patients with coronary artery disease. All patients approached before surgery and the main investigator (ZM) collected the data. All participants were asked to complete the study questionnaires in a calm setting. In the case of illiterate individuals, the main investigator helped people to complete the questionnaires. In all instances completion of the questionnaires took about $15 \mathrm{~min}$. The inclusion criteria were as follows: suffering from CAD, minimum age of 18 years; Iranian; and willing to participate in the study and not suffering from hearing loss or any mental or cognitive disorders. This latter condition was examined by a self-report questionnaire. Patients with other disease conditions were not included. In addition all patients were asked to respond to a short demographic questionnaire including items on age, martial status, education, employment, income, smoking, and history of hospitalization. In Particular income was assessed based on the following single item: overall how would you describe your current income condition. The response category included as poor, intermediate, and good. This was based on similar studies conducted in Iran lending support to its validity [20].

\section{Sample size justification}

For the study purposes we thought 200 patients with coronary artery disease (10 participants per item) are needed for exploratory factor analysis (EFA) and 250 patients are needed for confirmatory factor analysis (CFA) $[21,22]$. However, in practice overall we recruited 500 patients with coronary artery disease who were hospitalized in cardiac departments of hospitals affiliated to medical schools in Tehran, Iran.

\section{The coronary artery disease education questionnaire (CADE-Q)}

The Coronary Artery Disease Education Questionnaire (CADE-Q) evaluates patients' knowledge about their disease and related factors. The questionnaire was developed following a general review of the literature, with feedback from cardiologists and a cardiac rehabilitation multidisciplinary team. The CADE-Q initially validated in Brazilian Portuguese and was confirmed to be a valid tool, with strong overall characteristics in terms of content, development and testing [13]. Then an English version of the questionnaire was developed including 19 items covering five areas of knowledge: pathophysiology, signals and symptoms of the disease; risk factors, prevention of risk factors (lifestyle habits); diagnosis, treatment and medicines; and exercise [Additional file 1]. Each item has four statements that according to knowledge levels, the following criteria will apply: most correct answers $=3$; somewhat correct answers $=1$; wrong answer $=0$; and don't know $=0$. The sum of scores could be computed to calculate the knowledge score [23].

\section{Translation procedure}

Forward-backward translation procedure was used to translate the English version of the questionnaire into 
Persian. As such, two independent professionals translated the questionnaire from English into Persian. Then a consolidated Persian version of the two translations was provided with the best translation available. Subsequently two experts back translated the Persian version into English and it was compared with the original English version by the research team and the provisional version of the Persian version was provided. In order to examine content validity, 10 experts (two cardiologists, five assistant professors in nursing, and three assistant professors experienced in questionnaire design) were asked to qualitatively examine the questionnaire, and provide their opinions on the questionnaire in terms of grammar, vocabulary, necessity, importance, placement of the words, and scoring. The experts made no changes to the questionnaire. Then, the CADE-Q was administered to 10 patients with coronary artery disease who met the inclusion criteria with maximum variance in order to assess the face validity of the questionnaire. Their views on appropriateness, difficulty, relevancy and ambiguity of the items were assessed. Almost all patients did not indicate any problems and thus the questionnaire was made ready for psychometric evaluation.

\section{Statistical analysis}

The following analyses were performed in order to assess the psychometric properties of the questionnaire:

\section{Item analysis}

Inter-item correlation was assessed in order to suggest if there was any potentially problematic item. It was expected that all correlations range between 0.3 and 0.7 [24].

2. Structural validity

(i) The exploratory factor analysis (EFA) was performed to extract latent factors. The KaiserMeyer-Olkin (KMO) test for sampling adequacy and the Bartlett's test for sphericity were used. The KMO values between 0.7 and 0.8 are consider to be as good, and values between 0.8 and 0.9 are consider as excellent [25]. Then, the latent factors were extracted using the maximum likelihood estimation, the promax rotation, and scree plots. Presence of each item in the factor was determined according to communalities of above 0.3 in the EFA [26].

(ii) In the second step, the factors extracted using the first- and second-order factor analysis and the most common goodness of-fit indices of the proposed model were assessed based on the threshold of acceptance and the maximum likelihood approach. There are no golden rules for evaluating goodness-of-fit indices; however, it is necessary to report a variety of indices because different indices often reflect different features of the model [27]. Fit indices employed in the study included Chisquare ( $\mathrm{x}$ ), Chi-square/degree-of-freedom ratio (normalized Chi-square CMIN/DF), Adjusted Goodness-of-Fit Index (AGFI), Parsimonious Comparative Fit Index (PCFI), Comparative Fit Index (CFI), Incremental Fit Index (IFI), Parsimonious Normed Fit Index (PNFI), Root Mean Square Error of Approximation (RMSEA) [28]. In the second-order factor analysis, it was assumed that the extracted latent variables in the first stage were present. Thus, the second-order factor

Table 1 Demographic Characteristics of the study sample $(n=500)$

\begin{tabular}{|c|c|c|}
\hline Gender & Number & $\%$ \\
\hline Male & 285 & 57 \\
\hline Female & 215 & 43 \\
\hline \multicolumn{3}{|c|}{ Age group (years) } \\
\hline $21-40$ & 97 & 19.4 \\
\hline $41-60$ & 246 & 49.2 \\
\hline $61-80$ & 139 & 27.8 \\
\hline$>80$ & 18 & 3.6 \\
\hline \multicolumn{3}{|l|}{ Marital status } \\
\hline Married & 369 & 73.8 \\
\hline Single & 46 & 9.2 \\
\hline Widow & 74 & 14.8 \\
\hline Divorced & 11 & 2.2 \\
\hline \multicolumn{3}{|c|}{ Employment status } \\
\hline Housewife & 184 & 36.8 \\
\hline Employed & 173 & 34.6 \\
\hline Unemployed & 45 & 9 \\
\hline Retired & 98 & 19.6 \\
\hline \multicolumn{3}{|c|}{ Educational level } \\
\hline Illiterate & 114 & 22.8 \\
\hline Primary & 119 & 23.8 \\
\hline Secondary & 181 & 36.2 \\
\hline Higher & 86 & 17.2 \\
\hline \multicolumn{3}{|c|}{ History of smoking } \\
\hline Yes & 83 & 16.6 \\
\hline No & 417 & 83.4 \\
\hline \multicolumn{3}{|l|}{ Income } \\
\hline Poor & 123 & 24.6 \\
\hline Intermediate & 292 & 58.4 \\
\hline Good & 85 & 17 \\
\hline \multicolumn{3}{|c|}{ Hospitalization history (in the last year) } \\
\hline Yes & 329 & 65.8 \\
\hline No & 171 & 34.2 \\
\hline
\end{tabular}


analysis represented the more general concepts at secondary and upper levels [29].

(iii) Convergent and divergent validity were assessed using the average variance extracted (AVE), the maximum shared squared variance (MSV), and the average shared squared variance (ASV) The convergent validity to be stablished, the AVE should be above 0.5 , and in order for the divergent validity to be stablished, the ASV and the MSV should be lower than the AVE [30].

(iv) Sensitivity was examined by extracting factor loadings for patients with or without a history of the hospitalization. It was expected to achieve similar factors for both groups .

3. Reliability assessment

(i) In order to assess the internal consistency of the CADE-Q, the Cronbach's alpha coefficient was estimated first for the whole questionnaire and then for each extracted factor. An alpha value above 0.7 was considered to indicate good internal consistency [31].

(ii) Stability was assessed using the intraclass correlation coefficient (ICC). When this index is above 0.75 , there is a good level of stability [32].

Table 2 The component matrix for items and total variance observed resulted from maximum likelihood analysis after promax rotation for the CADE-Q $(n=250)$

\begin{tabular}{|c|c|}
\hline Factor & Factor loading \\
\hline \multicolumn{2}{|l|}{ Lifestyle habits \& exercise } \\
\hline 19-Which interventions can extend and improve a patient's quality of life for persons recovering from a cardiac event? & 0.95 \\
\hline $\begin{array}{l}\text { 15-Which of the following changes in the body resulting from regular physical exercise are most important to long } \\
\text { term cardiac health? }\end{array}$ & 0.67 \\
\hline 18-Which of the statements below regarding psychological stress is most correct? & 0.62 \\
\hline 14-Guidelines for Physical Activity for people with coronary disease should be based upon which of the following: & 0.58 \\
\hline 13-Based on your knowledge about exercise and CAD, choose the most appropriate statement below: & 0.55 \\
\hline 4- Which of the following statements is most accurate regarding our understanding of CAD? & 0.49 \\
\hline 16-Which of the following statements best describes the pattern for exercise activity in persons recovering from a heart event: & 0.47 \\
\hline Eigenevalue & 4.32 \\
\hline$\%$ variance & 26.35 \\
\hline \multicolumn{2}{|l|}{ Risk factors } \\
\hline 3-Which description below is a typical symptom of CAD & 0.47 \\
\hline 2-Which factors have the most influence on the risk of myocardial infarction. & 0.45 \\
\hline 6-Of the investigations listed below, which ones provide the most precise information about the diagnosis and prognosis of CAD? & 0.36 \\
\hline 5-The best time of the day for people with coronary disease to carry out their prescribed exercise is: & 0.31 \\
\hline 7-Which of the following statements about the management of blood cholesterol levels is most accurate? & 0.30 \\
\hline Eigenevalue & 1.95 \\
\hline$\%$ variance & 4.92 \\
\hline \multicolumn{2}{|l|}{ Diagnosis and treatment } \\
\hline 1-Coronary Artery Disease (CAD) is: & 0.75 \\
\hline $\begin{array}{l}\text { 17-Which of the following statements is the most appropriate guidance around levels of blood pressure levels in } \\
\text { persons with CAD: }\end{array}$ & 0.53 \\
\hline $\begin{array}{l}\text { 10-Which values for LDL cholesterol and HDL cholesterol are the optimal targets persons with established CAD } \\
\text { (values in mmol/litre)? }\end{array}$ & 0.39 \\
\hline 11-In which of the following situations would you avoid carrying out your regular physical exercise? & 0.36 \\
\hline Eigenevalue & 3.18 \\
\hline$\%$ variance & 2.67 \\
\hline \multicolumn{2}{|l|}{ Signals \& symptoms and medicine } \\
\hline 12-While walking, if you experience a new episode of severe chest discomfort that you think that is angina, you should: & 0.38 \\
\hline 8-Which of the following statements about the use of "nitroglycerin" is most accurate & 0.30 \\
\hline Eigenevalue & 2.24 \\
\hline$\%$ variance & 2.15 \\
\hline
\end{tabular}




\section{Normal distribution of data}

The normal distribution of the data, the outliers, and missing data were separately assessed. The presence of multivariate outliers was assessed using the Mahalanobis d-squared method $(p<0.001)$ and the violation of multivariate kurtosis using the Mardia coefficient (>8) [33] The number of missing data was assessed using multiple imputation analysis, which was then replaced with participants' mean responses. Data analysis was carried out using SPSS 16.0 (SPSS Inc., Chicago, Illinois) and AMOS 17.0 (SPSS Inc., Chicago, Illinois) statistical packages.

\section{Results}

Patients' characteristics

In all 500 patients with coronary artery disease were approached and all agreed to took part in the study. Of these $285(57 \%)$ were male, $73.8 .0 \%(n=369)$ married, and the mean age of participant was $53.6(\mathrm{SD}=14.36)$ years, and indicated themselves as having intermediate economic status (58.4\%). Most participants reported that they had been hospitalized at least once in the last year $(65.8$ \%). The characteristics of the participants are shown in Table 1.

\section{Item analysis}

The inter-item correlation was estimated among items and a satisfactory results obtained. The results showed a positive and significant correlation between all items [Additional file 2].

\section{Exploratory factor analysis}

The sampling adequacy (KMO) was calculated as 0.842 and Bartlett's test was calculated as $(p<0.001)$. The EFA resulted in the extraction of four factors, which explained $48.9 \%$ of the total variance (Table 2 ).

\section{Confirmatory factor analysis}

The results of the Chi-square test for goodness-of-fit were first obtained and other indices were then assessed for the fit of the model. According to Table 3, all the indices confirmed a good fit of the final model. According to the final factor structure of the CADE-Q, correlations between the measurement errors of items 2 and 3, 18 and 19 were observed (Fig. 1).

Following the first-order CFA, a separate assessment of the factors of the CADE-Q and the correlation between the constructs occurred. Subscales were determined using structural equations. The second-order factor analysis was performed to examine whether or not all the factors fitted the general concept of "knowledge." Table 3 presents the indices of fit for the secondorder CFA compared to the first-order model. Figure 2 shows the structural model and the CFA of the CADE-
Table 3 The range of acceptable fit indexes for confirmatory factor analysis

\begin{tabular}{llll}
\hline Indexes* & Cut off values & First order & Second order \\
\hline CMIN/df $^{*}$ & $<3$ & 2.08 & 2.08 \\
$P$ value & & 0.0001 & 0.0001 \\
GFI & $\geq 0.05$ & 0.96 & 0.96 \\
AGFI & $\geq 0.90$ & 0.94 & 0.94 \\
RFI & $\geq 0.90$ & 0.91 & 0.91 \\
TLI & $\geq 0.90$ & 0.94 & 0.94 \\
IFI & $\geq 0.95$ & 0.96 & 0.96 \\
CFI & $\geq 0.90$ & 0.96 & 0.96 \\
PCFI & $\geq 0.5$ & 0.70 & 0.70 \\
PNFI & $\geq 0.5$ & 0.70 & 0.73 \\
RMSEA & $\leq 0.08$ & 0.04 & 0.04
\end{tabular}

* Abbriviations: CMIN/DF Minimum Discrepanscy Funcation by degrees of freedom divided, GFI Good of Fit Index, AGFI Adjusted Good of Fit Index, RFI Relative Fit Index, TLI Tucker -Lewis Index, IFI Incremental Fit Index, CFI Comparative Fit Index, PCFI Parsimonious Comparative Fit Index, PNFI Parsimonious Normed Fit Index, RMSEA Root Mean Square Error of Approximation

** Derived from chi-square test $\left(X^{2}\right)$, which is one of measures for fit indexes in structural equation modeling (SEM). A non-significant result for this test indicates good model fit (i.e., $p>.05$ )

Q with standardized factor loadings. The factor loadings were greater than 0.3 for all the items and were significant at $p<0.05$.

\section{Convergent and discriminant validity}

Assessing the AVE $(0.40,0.31,0.34)$, MSV $(0.67,0.64$, $0.67)$ and ASV $(0.56,0.58,0.66)$, however, showed that the CADE-Q does not have a good convergent and divergent validity.

\section{Sensitivity analysis}

Sensitivity as assessed by exploratory factor analysis for patients with or without history of the hospitalization indicated similar factor extraction as expected [Additional file 3].

\section{Reliability}

Finally, a Cronbach's alpha of 0.84 ranging from 0.50 to 0.82 was found for the whole scale and the subscales, respectively. In addition, an ICC of 0.88 ranging from 0.78 to 0.87 was found for the whole scale and the subscales, respectively (Table 4).

\section{Discussion}

The present study was aimed to translate the Coronary Artery Disease Education Questionnaire (CADE-Q) into Persian and assess its validity and reliability to be used in epidemiological and clinical studies. We used rigorous methods based on both psychometric and conceptual criteria. The final CADE-Q was shorter than original one with improved fit indices relative to the long 


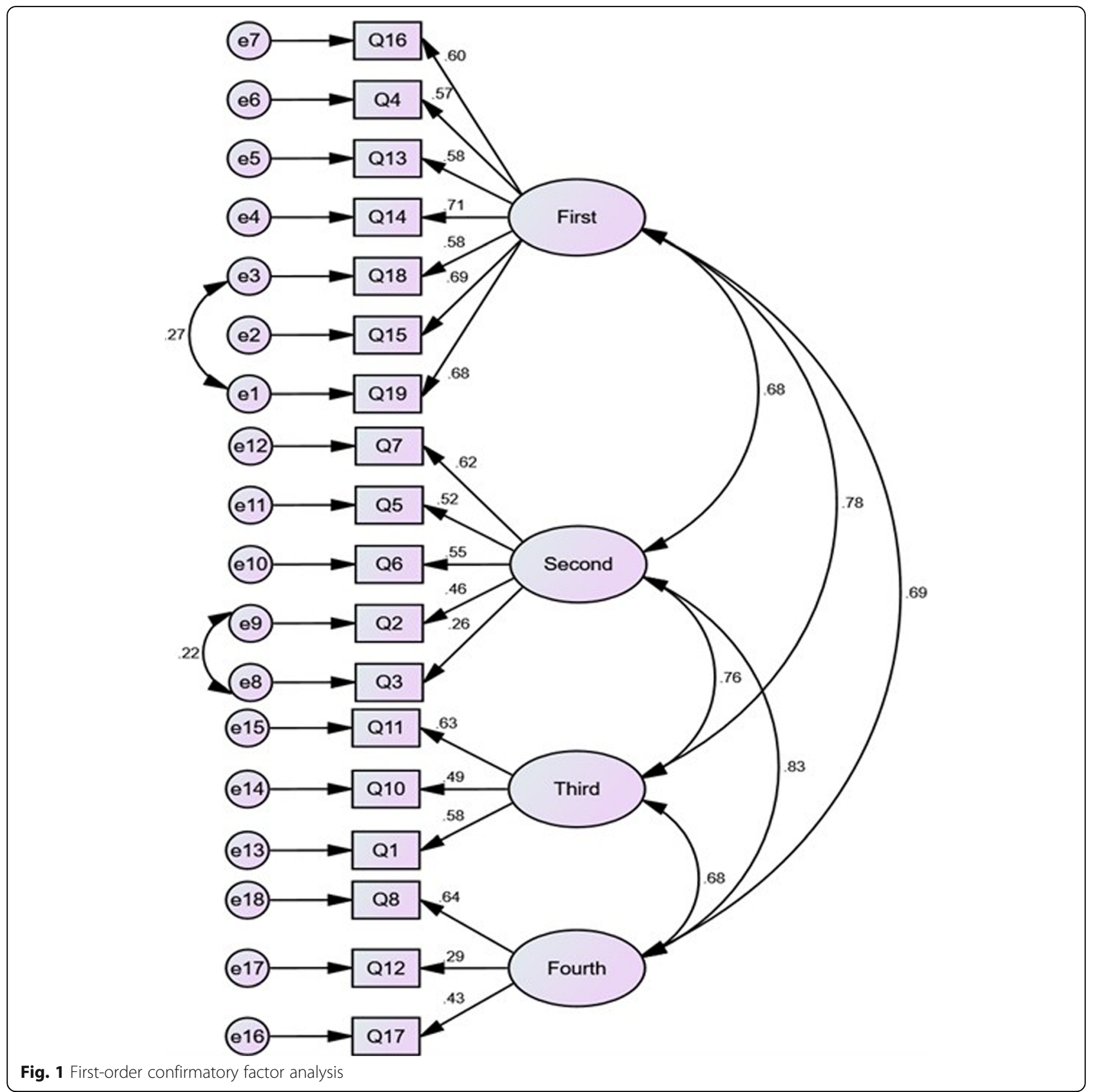

version. However, we kept the English version consistent with the original conceptual model.. It covers the key dimensions of the 'lifestyle habits \& exercise', 'risk factors' and 'diagnosis and treatment'. In addition, reliability, in terms of internal consistency, was preserved in the Persian version. However, one should note that we reduced five dimensions to three dimensions that, to some extent, is not unusual. To explain the issue further it is necessary to acknowledge that there are three CADE-Q versions: one consisting of five dimensions with 19 items (CADE-Q) [23], and the second version containing four dimensions with 31 items (CADE-QII)(18) and the third that is the short version containing four dimensions with 20 items (CADE-Q SV) [19]. Although there were a good similarity between the Persian version and the English version, the difference on number of dimensions might relate to difference in culture and environment. Further investigations might shed more light on this issue.

The first factor identified in the CADEQ includes a general viewpoint of lifestyle and exercise. The exercise concept is broadly about how a person measures the concept of having physical activity in different aspects of his or her life. This factor suggests that the first step in 


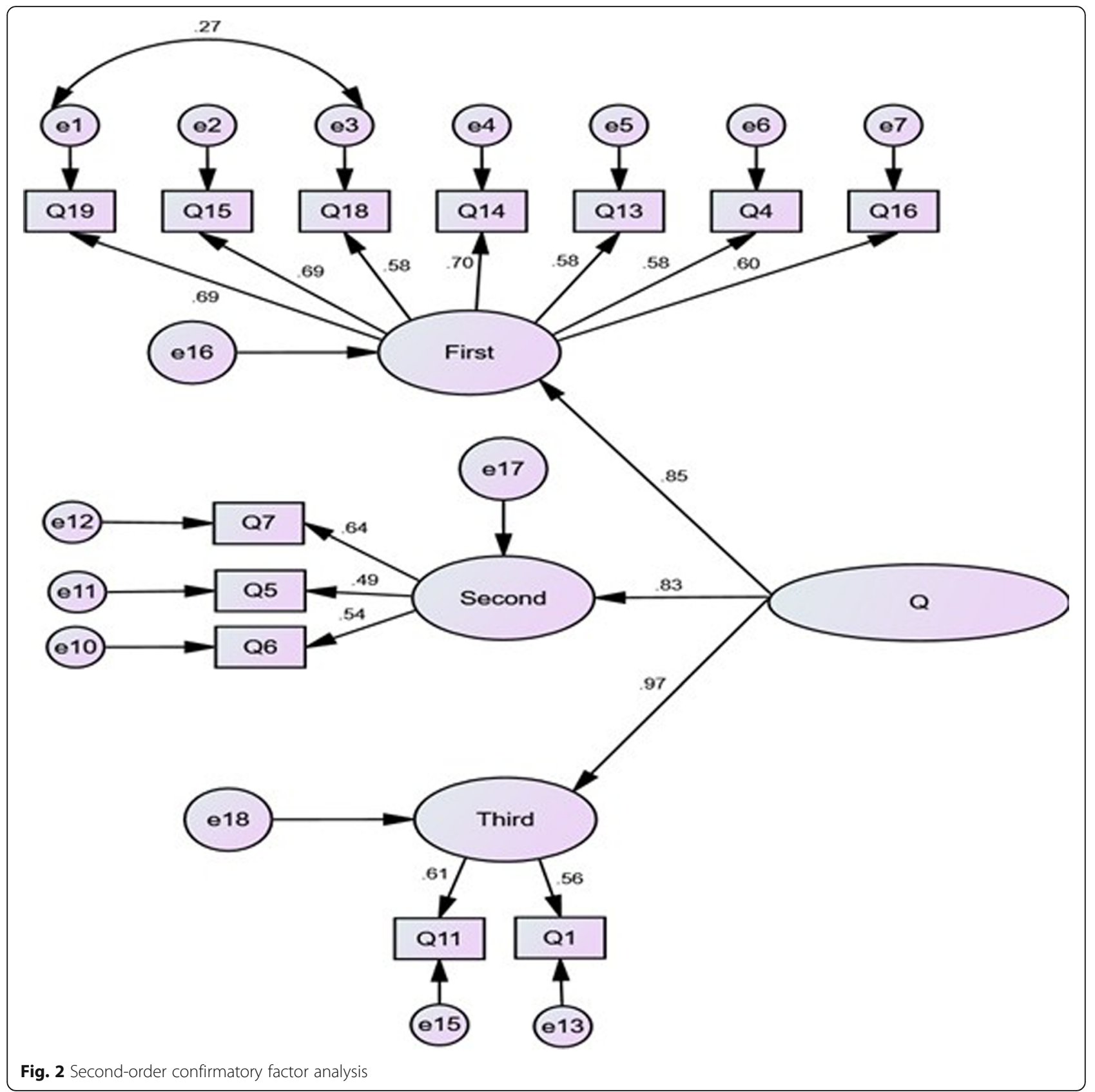

Table 4 The Cronbach's alpha and the Intraclass Correlation Coefficients (ICC) for the Persian version of the CADE-Q

\begin{tabular}{lll}
\hline Dimension & Cronbach's a & ICC $(\boldsymbol{n}=100)$ \\
\hline Lifestyle habits \& exercise & 0.825 & 0.870 \\
Risk factors & 0.553 & 0.782 \\
Diagnosis and treatment & 0.507 & 0.825 \\
Total score & 0.844 & 0.886 \\
\hline
\end{tabular}

maintaining heart health is to establish a good relationship with health care providers. However, making permanent changes requires learning a series of things, which include heart disease and the types of behaviors and conditions that can increase the risk in a person [34].

The results of EFA showed that the second dimension of CADEQ questionnaire relates the risk factors. Therefore, the best solution includes timely investigation, diagnosis, control of risk factors, and prevention of cardiovascular disease. Making healthy lifestyle changes based on guidelines obtained from decades of research not only prevents cardiovascular diseases, but also 
reduces the risk of dangerous diseases such as cancer and diabetes [35].

The third factor extracted in the CADEQ questionnaire includes the diagnosis and treatment dimension. Physicians believe that diagnostic tools and methods are very important to evaluate cardiac function for the diagnosis, treatment and follow-up, and have a significant effect on life expectancy and improving the quality of life of patients with coronary artery disease [36].

The fourth factor extracted in the CADEQ relates to the signals \& symptoms and medicine. It is important to note that the signals and symptoms of the disease are the most important diagnostic tools in all diseases, including heart disease, and the physician uses diagnostic methods to confirm and prove clinical findings, or complete the required information based on the patient's history and careful examinations. Therefore, patients with coronary artery disease should be fully aware of this important issue [37].

To find a more precise model of structural equations, the second-order CFA was also performed. This method seeks to obtain a more significant method of data collection while assuming that the latent variables in the common variance are due to one or more higher-order factors and that the intended scale has two orders [29]. A high correlation between the first-order constructs shows that the latent variables do not fully act as an independent variable and the correlation between them reflects the presence of a more general construct (knowledge) in a secondary conceptual level, where the best approach to the assessment of the structure is structural equation modeling, since it can identify the first-order constructs that were proposed as the latent variables [30] Anderson and Gerbing proposed that the intended construct must first be created through first-order factor analysis, and the good fit of the conceptual construct be then determined for the assessment of the structural equation model using second-order factor analysis [38].

Another finding of the present research showed that this scale has a good internal consistency. In this research, the Cronbach's alpha coefficients of the questionnaire were in the range of $0.577-0.825$ and the total Cronbach's alpha coefficients of the questionnaire was 844; however, the Cronbach's alpha coefficients was reported to be $0.76-0.94$ in Gisis's study that may be due to the fact that no question has been removed from the above questionnaire in this study [19]. It is worth noting that the Cronbach's alpha coefficients was expressed in the range of $0.66-0.71$ in the second version of the CADEQ questionnaire, which is relatively similar to our study [39]. The reliability of the questionnaire was also evaluated using a test-retest method. The results showed a good stability for the aforementioned questionnaire. The ICC value is between 0.882 and 0.886 .

\section{Limitations}

This study had a number of limitations, including the use of the self-report method of data collection, which can entail errors in reporting. Also, since the study was conducted in a certain geographical region, generalizing the results to wider geographical areas should be pursued with care. Future research should consider in different geographical regions.

\section{Conclusion}

Overall, the findings of this study suggest that CADEQ is acceptable in terms of psychometric properties. The questionnaire is easy to use and can be completed both by a patient or healthcare provider in a variety of settings.

\section{Supplementary information}

Supplementary information accompanies this paper at https://doi.org/10. 1186/s12872-020-01628-5.

Additional file 1. The Coronary Artery Disease Education Questionnaire (CADE-Q).

Additional file 2. Inter-item correlations for the CADE-Q.

Additional file 3. The EFA for the CADE-Q among patients with and without hospitalization history.

\begin{abstract}
Abbreviations
AGFI: Adjusted goodness of fit index; AVE: Average variance extracted; ASV: Average shared variance; CFA: Confirmatory factor analysis;

CFI: Comparative fit index; CMIN/DF: Minimum Discrepanscy Funcation by degrees of freedom divided; EFA: Exploratory factor analysis; GFI: Goodness of fit index; ICC: Intraclass correlation coefficients; IFI: Incremental fit index; KMO: Kaiser-Meyer-Olkin; MSV: Maximum variance; PCFI: Parsimonious comparative fit index; PNFI: Parsimonious normed fit index; RMSEA: Root mean square error of approximation; SEM: Structural equation modeling; SRMR: Standardized root mean square residual; TLI: Tucker -Lewis index
\end{abstract}

\section{Acknowledgments}

We thank all participants who made this study possible.

Authors' contributions

MHK was the study supervisor and contributed to all aspect of the study. ZM was data collection; RB was the main investigator and provided the first draft. AM and NR was the study advisor and contributed to the study design, $A M$ and RB critically reviewed the paper and provided the final draft. RB was the statistical advisor and contributed to data analysis. All authors read and approved of the final manuscript.

\section{Funding}

None.

Availability of data and materials

The datasets are available from the corresponding authors on request.

Ethics approval and consent to participate

Shahed University Ethics Committee approved the study. All participants signed informed consent form.

Consent for publication

Not applicable.

Competing interests

The authors declare that they have no competing interests. 


\section{Author details}

${ }^{1}$ Faculty of Nursing \& Midwifery, Shahed University, Tehran, Iran. ${ }^{2}$ Social Determinants of Health Research Center, Semnan University of Medical Sciences, Semnan, Iran. ${ }^{3}$ Elderly Care Research Center, Faculty of Nursing \& Midwifery, Shahed University, Tehran, Iran. ${ }^{4}$ Health Metrics Research Center, Iranian Institute for Health Sciences Research, ACECR, Tehran, Iran. ${ }^{5}$ Faculty of Humanity Sciences, University of Science \& Culture, ACECR, Tehran, Iran.

Received: 13 July 2019 Accepted: 17 July 2020

Published online: 23 July 2020

\section{References}

1. Mampuya WM. Cardiac rehabilitation past, present and future: an overview. Cardiovasc Diagn Ther. 2012;2(1):38.

2. Mendell J, Bates J, Banner-Lukaris D, Horvat D, Kang B, Singer J, et al. What do patients talk about? A qualitative analysis of online chat sessions with health care specialists during a "virtual" cardiac rehabilitation program. Telemed J E Health. 2019;25(1):71-8. https://doi.org/10.1089/tmj.2017.0206.

3. Hafiz AM, Poulin MF. A hybrid model for advanced structural heart disease training programs: the attending-fellow-in-training model. J Am Coll Cardiol. 2018;71(7):802-7. https://doi.org/10.1016/j.jacc.2018.01.008.

4. Modica M, Castiglioni P, Minotti A, Faini A, Racca V, Ferratini M. Psychological Profile in Coronary Artery By-Pass Graft Patients vs. Valve Replacement Patients Entering Cardiac Rehabilitation after Surgery. Sci Rep. 2018;8(1):14381

5. Korzeniowska-Kubacka I, Bilińska M, Piotrowska D, Wolszakiewicz J, Piotrowicz R. Impact of exercise-based cardiac rehabilitation on attitude to the therapy, aims in life and professional work in patients after myocardial infarction. Med Pr. 2019;70(1):1-7. https://doi.org/10.13075/mp.5893.00701.

6. Hunt KJ, Resendez RG, Williams K, Haffner SM, Stern MP. National Cholesterol Education Program versus World Health Organization metabolic syndrome in relation to all-cause and cardiovascular mortality in the San Antonio heart study. Circulation. 2004;110(10):1251-7.

7. Boyde M, Turner C, Thompson DR, Stewart S. Educational interventions for patients with heart failure: a systematic review of randomized controlled trials. J Cardiovasc Nurs. 2011;26(4):E27-35.

8. Krumholz HM, Amatruda J, Smith GL, Mattera JA, Roumanis SA, Radford MJ, et al. Randomized trial of an education and support intervention to preventreadmission of patients with heart failure. J Am Coll Cardiol. 2002; 39(1):83-9.

9. Bertelsen JB, Dehbarez NT, Refsgaard J, Kanstrup H, Johnsen SP, Qvist I, et al. Shared care versus hospital-based cardiac rehabilitation: a cost-utility analysis based on a randomised controlled trial. Open Heart. 2018:5(1): e000584. https://doi.org/10.1136/openhrt-2016-000584.

10. Awad A, Al-Nafisi H. Public knowledge of cardiovascular disease and its risk factors in Kuwait: a cross-sectional survey. BMC Public Health. 2014;14(1) 1131.

11. Lee MBK. Psycho-social support for patients with cardiovascular disease (CVD): barriers to a cardiac rehabilitation program (CRP). Canada: Simon Faraser University; 2016.

12. GLdM G, Leite CM, Durieux A, IdC S, MSd A, MMd B, et al. Validação para o português do Maugerl CaRdiac preventiOn-Questionnaire (MICRO-Q). Arq Bras Cardiol. 2010;94(3):394-400.

13. GLdM G, RZd S, Schveitzer $\vee$, Barros AL, Recchia TL, Oh P, et al. Development and validation of the Brazilian Portuguese version of the cardiac rehabilitation barriers scale. Arq Bras Cardiol. 2012;98(4):344-52.

14. de Melo Ghisi GL, Oh P, Benetti M, Grace SL. Barriers to cardiac rehabilitation use in Canada versus Brazil. J Cardiopulmon Rehabil Prevent. 2013;33(3):173-9.

15. de Melo Ghisi GL, Grace SL, Thomas S, Evans MF, Sawula H, Oh P. Healthcare providers' awareness of the information needs of their cardiac rehabilitation patients throughout the program continuum. Patient Educ Couns. 2014:95(1):143-50.

16. Bonin CD, Santos RZ, Ghisi GL, Vieira AM, Amboni R, Benetti M. Construction and validation of a questionnaire about heart failure patients' knowledge of their disease. Arq Bras Cardiol. 2014;102(4):364-73. https://doi.org/10.5935/ abc.20140032.

17. Bonin CDB, RZd S, GLdM G, Vieira AM, Amboni R, Benetti M. Construction and validation of a questionnaire about heart failure patients' knowledge of their disease. Arq Bras Cardiol. 2014;102(4):364-73.
18. Ghisi GL, Grace SL, Thomas S, Evans MF, Oh P. Development and psychometric validation of the second version of the coronary artery disease education questionnaire (CADE-Q II). Patient Educ Couns. 2015;98(3): 378-83. https://doi.org/10.1016/j.pec.2014.11.019.

19. Ghisi GLM, Sandison N, Oh P. Development, pilot testing and psychometric validation of a short version of the coronary artery disease education questionnaire: the CADE-Q SV. Patient Educ Couns. 2016;99(3):443-7. https://doi.org/10.1016/j.pec.2015.11.002.

20. Salehi-Isfahani D. Poverty and income inequality in the Islamic Republic of Iran. Revue internationale des études du développement. 2017;(1):113-36. https://doi.org/10.3917/ried.229.0113.

21. Mundfrom DJ, Shaw DG, Ke TL. Minimum sample size recommendations for conducting factor analyses. Int J Test. 2005;5(2):159-68.

22. MacCallum RC, Widaman KF, Zhang S, Hong S. Sample size in factor analysis. Psychol Methods. 1999;4(1):84

23. de Melo Ghisi GL, Oh P, Thomas S, Benetti M. Development and validation of an English version of the coronary artery disease education questionnaire (CADE-Q). Eur J Prev Cardiol. 2013;20(2):291-300.

24. Polit DF, Yang F. Measurement and the measurement of change: A primer for the health professions. Philadelphia: Lippincott Williams \& Wilkins; 2015.

25. Bandari R, Heravi-Karimooi M, Miremadi M, Mohebbi L, Montazeri A. The Iranian version of geriatric anxiety inventory (GAI-P): a validation study. Health Qual Life Outcomes. 2019;17(1):118. https://doi.org/10.1186/s12955019-1176-z.

26. Miremadi M, Bandari R, Heravi-Karimooi M, Rejeh N, Sharif Nia H, Montazeri A. The Persian short form aging perceptions questionnaire (APQ-P): A validation study. Health Qual Life Outcomes. 2020;18(1):7. https://doi.org/10. 1186/s12955-019-1259-x.

27. Hoyle RH. Structural equation modeling: concepts, issues, and applications. United States: SAGE Publications; 1995.

28. Meyers LS, Gamst G, Guarino AJ. Applied multivariate research: design and interpretation. United States: SAGE Publications; 2012.

29. Gatignon H. Statistical analysis of management data. 3rd ed. New York: Springer science business media; 2014.

30. Hair J, Anderson R, Black B. Babin B. Pearson Education: Multivariate Data Analysis; 2016

31. Mayers A. Introduction to statistics and SPSS in psychology: Pearson education limited; 2013

32. Shoukri MM. Measures of Interobserver agreement and reliability. United States: CRC Press; 2011.

33. Esposito Vinzi V, Chin WW, Henseler J, Wang H. Handbook of partial least squares: concepts, methods and applications: Heidelberg, Dordrecht, London, New York: Springer; 2010.

34. Levy AE, Huang C, Huang A, Ho PM. Recent approaches to improve medication adherence in patients with coronary heart disease: Progress towards a learning healthcare system. Curr Atheroscler Rep. 2018:20(1):5.

35. Potvin L, Richard L, Edwards AC. Knowledge of cardiovascular disease risk factors among the Canadian population: Relationships with indicators of socioeconomic status. CMAJ. 2000;162:S5-11.

36. Salvado-Hernandez C, Cosculluela-Torres P, Blanes-Monllor C, ParelladaEsquius N, Mendez-Galeano C, Maroto-Villanova N, et al. [Heart failure in primary care: Attitudes, knowledge and self-care]. Aten Primaria. 2017.doi: https://doi.org/10.1016/j.aprim.017.03.008

37. de Melo Ghisi GL, Britto R, Motamedi N, Grace SL. Disease-related knowledge in cardiac rehabilitation enrollees: correlates and changes. Patient Educ Couns. 2015;98(4):533-9

38. Anderson JC, Gerbing DW. Structural equation modeling in practice: A review and recommended two-step approach. Psychol Bull. 1988;103(3):411.

39. Ghisi GL, Durieux A, Manfroi WC, Herdy AH, Carvalho T, Andrade A, et al. Construction and validation of the CADE-Q for patient education in cardiac rehabilitation programs. Arq Bras Cardiol. 2010;94(6):813-22. https://doi.org/ 10.1590/s0066-782×2010005000045

\section{Publisher's Note}

Springer Nature remains neutral with regard to jurisdictional claims in published maps and institutional affiliations. 